\title{
Research on High Pressure Resistant Linear Variable Differential Transformer with Magnetic Ring
}

\author{
LI Ruifeng \\ College of Energy and Power Engineering, Lanzhou \\ University of Technology \\ Research Center for Hydraulics and Pneumatics \\ Engineering of Gansu Province \\ Lanzhou, China \\ e-mail:liruifengggdyx@163.com
}

\author{
JI Hong \\ College of Energy and Power Engineering, Lanzhou \\ University of Technology \\ Research Center for Hydraulics and Pneumatics \\ Engineering of Gansu Province \\ Lanzhou,China \\ e-mail:jihong@lut.cn
}

\author{
LI Xiaoping, SU Maliang \\ College of Energy and Power Engineering, Lanzhou \\ University of Technology \\ Research Center for Hydraulics and Pneumatics \\ Engineering of Gansu Province \\ Lanzhou,China \\ e-mail:1xp13669357016@163.com,smljqlut@163.com
}

\author{
XIE Hao, ZHANG Shuowen \\ College of Energy and Power Engineering, Lanzhou \\ University of Technology \\ Research Center for Hydraulics and Pneumatics \\ Engineering of Gansu Province \\ Lanzhou,China \\ e-mail:xiehaoorange@163.com, \\ 18919892115@163.com
}

\begin{abstract}
Aiming at the problems of traditional linear variable differential transformer (LVDT) with poor linearity and small stroke length ratio, a new kind of structure with magnetic ring was presented. The finite element simulation models of the before and after improved LVDT were established. The structural static analysis of pressure pipe was simulated by ANSYS to study the pressure capacity. For researching the effect of magnetic ring on the characteristics of LVDT, the transient electromagnetic field was simulated by Ansoft. The results show that the maximum deformation of pressure pipe appears at the unfixed end and the value is $2.7 \mu \mathrm{m}$ at 30MPa. After the magnetic ring is added in the bobbin, the LVDT's magnetic field distribute is greatly improved. Comparing with the unimproved LVDT's characteristics, the linearity of improved LVDT increases 5.6 times, the stroke length ratio improves 2.5 times and the sensitivity has a slight reduction. So the global Characteristics of LVDT is promoted a lot.

Keywords: LVDT; Magnetic ring; Linearity; Sensitivity;
\end{abstract} Stroke length ratio

\section{INTRODUCTION}

Linear variable differential transformer is used to measure the displacement based on the principle of electromagnetic induction. And it is the major installation of measuring the displacement and achieving close-loop control in the hydraulic field because of its good linearity, absolute reading and contactless measurement. At the present, the hydraulic components are developing towards to the direction of strong, small and smart. So a LVDT with high pressure resistant, small volume and high precision is highly necessary. So it is significant to research its abilities of pressure resistant, stroke length ratio and linearity.
In recent years, many scholars have carried out the research for LVDT. LIU Zhicai researched on the influence of structure parameters on the linearity and sensitivity by simulation [1]. REN Haiyan analyzed the influence of windings on the LVDT's property by using the magnetic simulation [2]. A parametric modeling method for electromagnetic simulation was presented based on the script template by LIU Ping [3]. A new concept was proposed by Ara K to compensate sensitivity changes caused by the variations of temperature and excitation conditions [4]. Das S used two-stage functional link artificial neural network to enhance the linearity of LVDT [5]. The influence of external magnetic on LVDT's linearity and sensitivity was studied by Masi A, Martino M [6-7]. The impact of different soft magnetic materials on the linearity and sensitivity of LVDT was researched by R. Yañez-Valdez[8]. Exploration to replace the core material by an encapsulated ferrofluid was done by Félix M [9].

The aim of this work is to improve the structure of LVDT to improve its characteristic.

\section{THE STRUCTURE AND WORKING PRINCIPLE OF LVDT}

\section{A. The Structure of LVDT}

The unimproved structure of LVDT is shown in fig.1.It comprises one primary winding; two secondary windings, symmetrically spaced with respect to the primary one to improve LVDT's linearity and sensitivity and enlarge the range of linearity; a movable core; a bobbin; two magnetic end plates; a magnetic shell; and a pressure pipe. The primary winding is excited by a sine wave. Magnetic end plates and shell not only improve LVDT's linearity and sensitivity, but also close the magnetic circuit [6]. 


\section{B. The Working Principle of LVDT}

The equivalent circuit diagram of LVDT is shown in fig.2. According to the electromagnetic induction principle, the differential voltage effective value of LVDT is

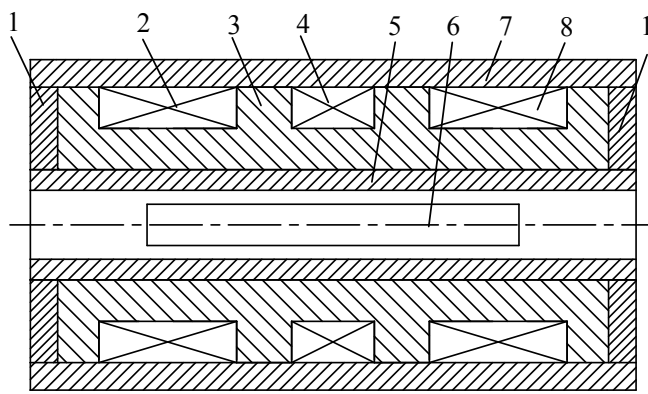

1-magnetic plate; 2-sencondary winding1; 3-bobbin; 4-primary winding; 5-pressurepipe; 6-core;

7-magnetic shell; 8 -secondary winding 2

Figure 1. The structure diagram of unimproved LVDT

$$
U_{O}=\frac{\omega\left(M_{1}-M_{2}\right) U_{I}}{\sqrt{R_{1}^{2}+\left(\omega L_{1}\right)^{2}}}
$$

When the core is in the middle, $M_{1}=M_{2}, U_{\mathrm{O}}=0$, the differential voltage effective value of LVDT is zero. When the core moves up, $M_{1}>M_{2}, U_{\mathrm{O}}>0$. When the core moves down, $M_{1}<M_{2}, U_{\mathrm{O}}<0$. With the movement of core, the mutual inductance between the primary winding and two secondary windings changes, which leads to the change of output voltage. Therefore the position of the core can be extracted by differential reading of the secondary signals. where

$$
\begin{array}{ll}
U_{\mathrm{I}} & \text { exciting voltage of primary winding } \\
U_{\mathrm{O}} & \begin{array}{l}
\text { differential output voltage } \\
R_{1}, R_{21}, R_{22}
\end{array} \\
& \begin{array}{l}
\text { resistant of primary winding and two } \\
\text { secondary windings }
\end{array}
\end{array}
$$

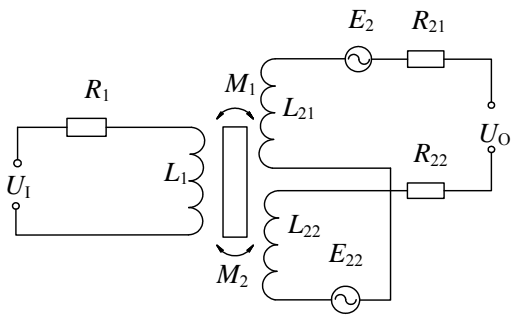

Figure 2. Equivalent circuit diagram of LVDT

$L_{1}, L_{21}, L_{22}$ self-inductance of primary winding and two secondary windings

$M_{1}, M_{2} \quad$ mutual inductance produced between the primary winding and two secondary windings

$E_{21}, E_{22} \quad$ induced electromotive force of two secondary windings

\section{The Improved Structure of LVDT}

As shown in fig.1, a pressure pipe is added in the bobbin to make sure the LVDT would work normally in the high pressure environment. The pressure pipe is nonmagnetic, so the magnetic field distribution is influenced and the optimization to magnetic field distribution by end tapes is waken. This structure has poor full-scale linearity and small stroke length ratio. To improve magnetic field distribution, linearity and increase stroke length ratio, a new kind of structure with magnetic ring was put forward. Two completely same magnetic rings being symmetrical about primary winding are designed in the bobbin. The improved structure is shown in fig.3. This structure has applied for a patent for invention (application No.: 201510184887.9).

\section{STRUCTURE STATIC ANALYSIS}

Pressure pipe is the pressure resistant component, which protects other components when the high pressure oil flows into LVDT. So the reliability of pressure pipe is very important to the LVDT's property. The structure

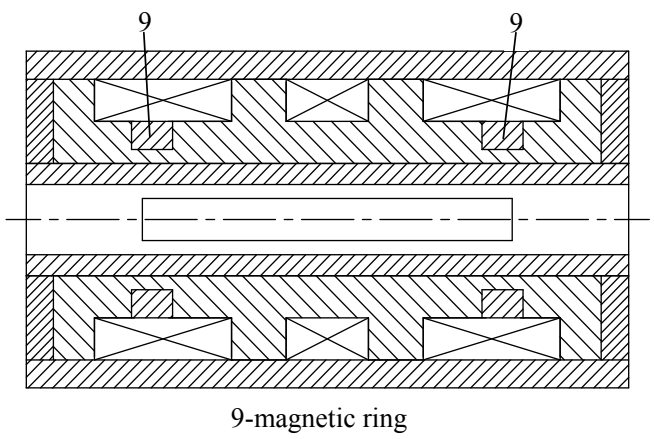

Figure 3. The structure diagram of improved LVDT

static analysis of pressure pipe was done by ANSYS Workbench.

\section{A. Materials Setting}

The pressure pipe's material is steel-stainless

\section{B. Loading}

In this paper, the reliability of pressure pipe is analyzed at $30 \mathrm{MPa}$. So $30 \mathrm{MPa}$ was loaded on the inner wall of pressure pipe.

\section{Constraints}

Under the normal working condition, LVDT is connected with measured components by pressure pipe. So the one end of pressure pipe is set as fixed constraints.

\section{Results Analysis}

The stress nephogram of pressure pipe is shown in fig.4. As shown in fig.4, the maximum deformation appears at the unfixed end and the value is $2.7 \mu \mathrm{m}$. Compared with the wall thickness $(1.5 \mathrm{~mm})$ of pressure pipe, it's very small. And it is far less than the gap between the pressure pipe and bobbin. So in the high pressure environment (less than $30 \mathrm{MPa}$ ), the structure will be satisfied with requirement of reliability.

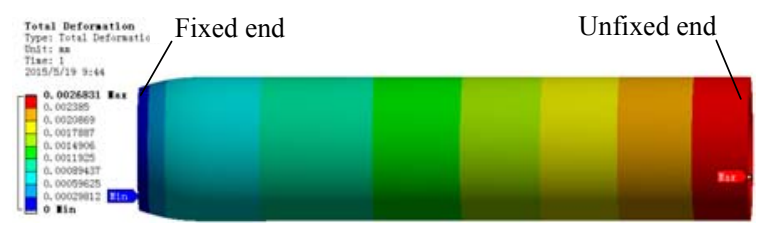

Figure 4. Stress nephogram of pressure pipe 


\section{TRANSIENT ELECTROMAGNETIC SIMULATION OF LVDT}

LVDT finite element simulation models before and after improved are established and simulated by Ansoft Maxwell to analyze the transient electromagnetic property.

\section{A. Establish Equivalent Simulation Model}

The model has been prepared in $2 \mathrm{D}$ environment. Taking into account the fact that the LVDT has cylindrical symmetry, half of the longitudinal section of the LVDT has been modeled (fig.5). And with the use of 2D model, the quantity of mesh can be decreased and the computational efficiency can be improved. Because the output property of LVDT is ultimately influenced by resistance and induction of coil rather than the section shape of coil, the circle section of coil is been equivalent to rectangular with same area to decrease the quantity of mesh and improve the computational efficiency.

\section{B. Define the Materials Property and Meshing}

Set material properties according to the table I. When it is meshed, comply with the following principles. The parts

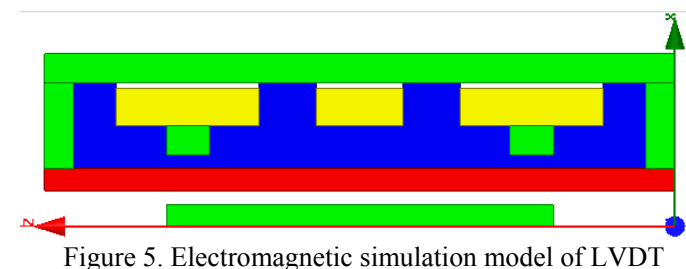

which have much influence on magnetic field distribution should be meshed densely, such as air gap and magnetic components. On the contrary, the parts have little influence on magnetic field distribution should be meshed sparsely. The mesh is shown in fig.6.

TABLE I. MATERIALS PROPERTY OF MODEL

\begin{tabular}{|c|c|c|}
\hline $\begin{array}{c}\text { Serial } \\
\text { number }\end{array}$ & Model & Materials \\
\hline \multicolumn{3}{|c|}{ 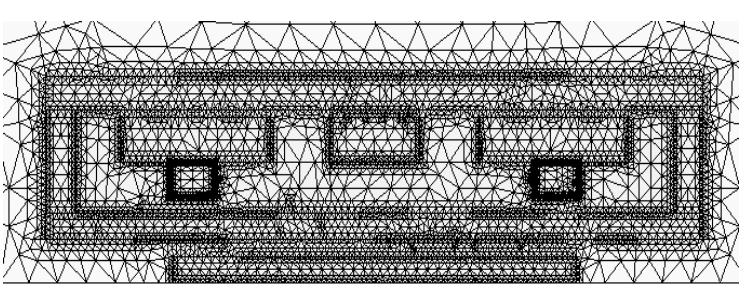 } \\
\hline \multicolumn{3}{|c|}{ Figure 6. The mesh of LVDT } \\
\hline 1 & Core & Magnetic materials \\
\hline 2 & Bobbin & Epoxy_Kevlar_xy \\
\hline 3 & Primary winding (564 turns) & Copper \\
\hline 4 & Secondary winding(948turns) & Copper \\
\hline 5 & End plate & Magnetic materials \\
\hline 6 & Shell & Magnetic materials \\
\hline 7 & Pressure pipe & Steel stainless \\
\hline 8 & Magnetic ring & Magnetic materials \\
\hline
\end{tabular}

\section{Exciting and Boundary Condition}

To gain the more accurate results, external exciting circuit edited by Maxwell Circuit Editor of Ansoft is employed. The circuit diagram is shown in fig.7. The exciting signal is Sinusoidal AC signal, whose frequency is $4 \mathrm{KHz}$ and amplitude is $2 \mathrm{~V}$.

Because the magnetic material will generate eddy effects in alternating field and the electrified coil have copper loss, eddy effects and copper loss are considered to
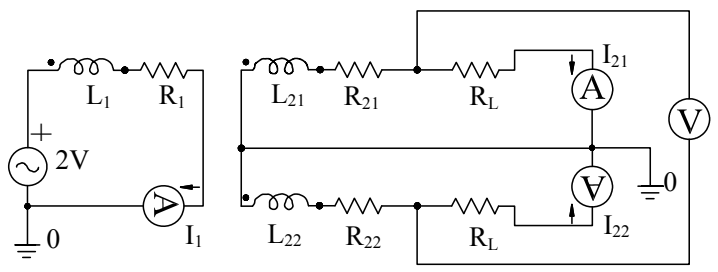

Figure 7. The external drive circuit of LVDT

ensure the accuracy of simulation results

Boundary condition is balloon.

\section{Simulation and Post-processing}

In this paper, the core position is going from $-6.5 \mathrm{~mm}$ to $+6.5 \mathrm{~mm}$ (when the core is in the middle, its position is 0 ). Due to the symmetrical structure of LVDT, the core position is going from 0 to $+6.5 \mathrm{~mm}$ in simulation. Ten points are taken to simulate at the range of $0 \sim 6.5 \mathrm{~mm}$. The step is $0.65 \mathrm{~mm}$.

In the process of simulation, computing time must be more than 3 cycles of exciting signal to ensure that the induced voltage of two secondary reaches stable. In this paper, the computing time is 10 cycles of exciting signal. The simulation curves are closer to the sine curve, the results are more accurate. So 100 points were taken to simulate in each cycle.

\section{E. Calculation Result and Analysis}

\section{1) Characteristic Curve}

The characteristic curve and corresponding fitting curve of before and after improved LVDT are shown in fig.8. In this paper, output characteristic curve which can directly reflect the variety of linearity and sensitivity is fitted by the least square method. As can be seen, when the core position is going from $3 \mathrm{~mm}$ to $6.5 \mathrm{~mm}$, the curve of LVDT without magnetic ring keeps parabolic growth. That is, the linearity is becoming poor. But the curve of LVDT with magnetic ring is keeping linear growth in the whole position. So the linearity of LVDT with magnetic rings is better than that without magnetic rings.

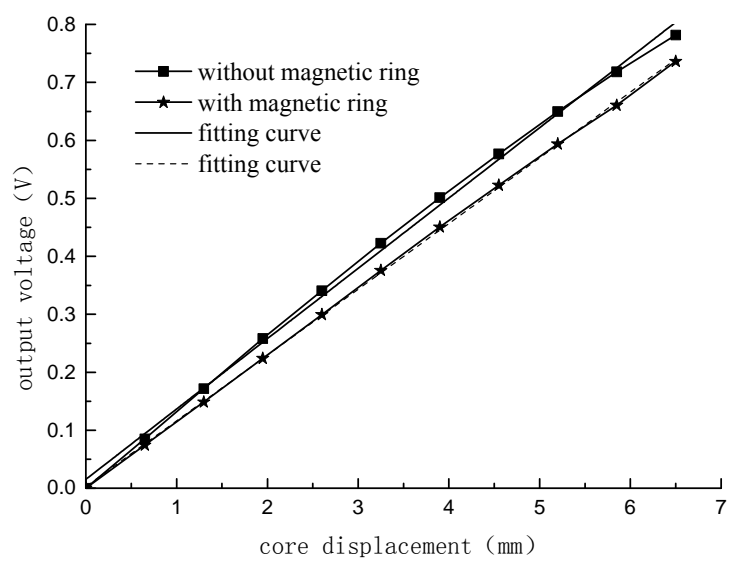

Figure 8. Characteristic curve of LVDT 


\section{2) Magnetic Field Distribution}

The magnetic field distribution of before and after improved LVDT is shown in fig.9. The core position is $3.25 \mathrm{~mm}$. According to the working principle of LVDT, when the core moves up from middle, the induced voltage of the secondary winding 1 increases gradually. Meanwhile, the induced voltage of secondary winding 2 decreases. The differential voltage of two secondary windings is output voltage of LVDT. When the core moves down the condition is similar.

The magnetic field intensity in the axial direction of solenoid remains relatively constant in the vicinity of middle and gradually reduces toward both sides [10]. And the variation of differential voltage incremental has the same principle with that of magnetic field intensity when the core is moving to end plate, as shown in fig.9a). That is the result that characteristics curve keeps parabolic growth. As shown in fig. 9b), two magnetic rings are magnetized. The magnetic field intensity at the end of LVDT is strengthened by two magnetic rings. So the differential output voltage substantially remains linear growth and the characteristic curve of LVDT keeps good linearity.

\section{3) Linearity}

Linearity is also called nonlinearity error. The smaller
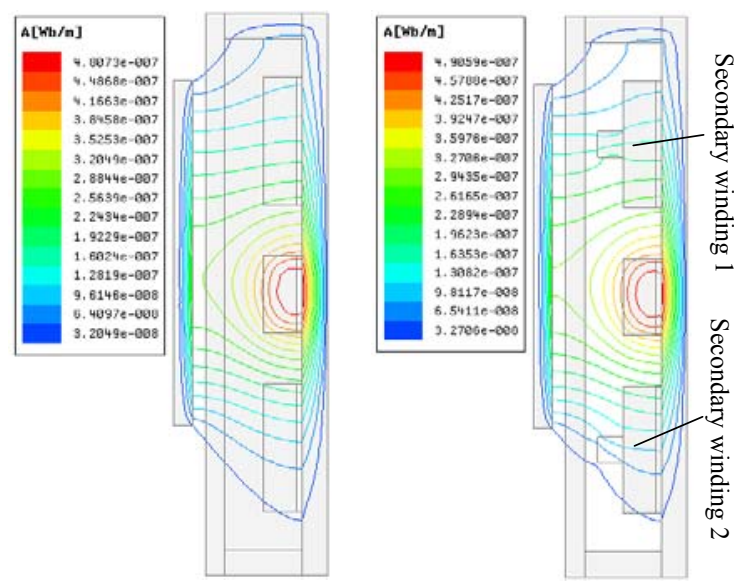

a) The magnetic field distribution b) The magnetic field distribution of unimproved LVDT of improved LVDT

Figure 9. Magnetic field distribution of LVDT

the nonlinearity error is, the better linearity is. The formula for calculation is as follow:

Where

$$
\gamma_{\mathrm{L}}=\frac{\Delta \mathrm{L}_{\max }}{y_{F S}} \times 100 \%
$$

$\Delta \mathrm{L}_{\max }$ The maximum deviation of the actual curve and fitting curve.

$\mathrm{y}_{\mathrm{FS}} \quad$ full scale output value.

Though (2), the linearity of original LVDT is $2.8 \%$ and the linearity of improved LVDT is $0.5 \%$. Therefore, the linearity of improvement increases 5.6 times comparing with the previous. Thus, the magnetic ring significantly improves the linearity of the LVDT.

\section{4) Sensitivity}

The formula for sensitivity calculation is as follow :

$$
S=\frac{\Delta y}{\Delta x}
$$

Where

$$
\Delta y \text { Incremental output. }
$$

$\Delta \mathrm{X}$ Incremental input.

The sensitivity of improvement is $113.4 \mathrm{mv} / \mathrm{mm}$ which decreases $7.9 \mathrm{mv} / \mathrm{mm}$ compared with that of original LVDT. While the reduction has a little influence on sensitivity.

\section{5) Stroke Length Ratio}

Stroke length ratio is the ratio between the effective linear stroke and total length of LVDT. Under the condition of obtaining same linear stroke, the larger stroke length ratio is, the smaller the volume is. The linear stroke of original LVDT is $\pm 2.6 \mathrm{~mm}$ and that of improvement is $\pm 6.5 \mathrm{~mm}$. They have same total length $(44 \mathrm{~mm})$. So the stroke length ratios of original LVDT and improvement are respectively 0.12 and 0.3 . The stroke length ratio improves 2.5 times after adding the magnetic ring. It shows that the magnetic ring has a larger influence on LVDT's stroke length ratio. This is very favorable to the miniaturization of LVDT.

\section{Conclusions}

A new kind of pressure resistant LVDT with magnetic ring was put forward. Compared with traditional LVDT, the volume has been decreased a lot and the linearity has been greatly improved, while other properties remain steady.

\section{ACKNOWLEDGMENT}

The authors would like to thank members of Research Center for Hydraulics and Pneumatics Engineering of Gansu Province for their useful discussions and suggestions.

\section{REFERENCES}

[1] LIU Zhi-cai. Digital signal processing algorithm and circuit research on LVDT displacement sensor [D]. Zhejiang: Zhejiang University Institute of Mechanical and Electronic Engineering and Control Engineering, 2012.

[2] Ren Hai-yan, LI Wen-zhang, ZHU Tin-wei, et al. The simulation modeling research of linear variable differential transformer displacement sensor [J]. Journal of Astronautic Metrology and Measurement, 2013,33(06):20-25.

[3] LIU Ping. Parametric simulation and optimization of linear variable differential transformer [D]. Xi'an University of Electronic Science and Technology,2010.

[4] Ara K. A differential transformer with temperature-and excitationindependent output[J]. Instrumentation and Measurement, IEEE Transactions on, 1972, 21(3): 249-255.

[5] Das S, Das D P, Behera S K. Enhancing the linearity of LVDT by two-stage functional link artificial neural network with high accuracy and precision[C]//Industrial Electronics and Applications (ICIEA), 2013 8th IEEE Conference on. IEEE, 2013: 1358-1363.

[6] Masi A, Danisi A, Losito R, et al. Study of magnetic interference on an LVDT: FEM modeling and experimental measurements[J]. Journal of Sensors, 2011, 2011 (529454): 1-9.

[7] Martino M, Danisi A, Losito R, et al. Design of a linear variable differential transformer with high rejection to external interfering magnetic field[J]. Magnetics, IEEE Transactions on, 2010, 46(2): 674-677.

[8] R. Yañez-Valdez, R. Alva-Gallegos, A. Caballero-Ruiz, L. RuizHuerta. Selection of soft magnetic core materials used on an LVDT prototype[J].Journal of Applied Research and 
Technology,2012,10(2):195-205.

[9] Félix M, Lizárraga A, Islas A, et al. Analysis of a ferrofluid core LVDT displacement sensor[C]//IECON 2010-36th Annual Conference on IEEE Industrial Electronics Society. IEEE, 2010: 1769-1772.

[10] JIA Rui-gao, XUE Qin-zhong. Electromagnetism [M].Beijjing: High Education Press, 2003. 